\title{
EVALUACIÓN DEL GASTO ECONÓMICO EN LA ATENCIÓN DE HIDATIDOSIS HUMANA EN JUNÍN, PERÚ
}

\author{
Raúl Montalvo ${ }^{1,2, a}$, Víctor Tiza ${ }^{3, b}$
}

\begin{abstract}
RESUMEN
El objetivo del presente estudio fue determinar la pérdida económica que ocasiona el tratamiento de la hidatidosis humana, para lo cual se realizó un análisis de costos de los casos reportados en la región Junín en Perú, durante el año 2013. El costo total (directo e indirecto) en dólares americanos por paciente atendido por el Ministerio de Salud (Minsa) fue USD 1 220,0; por el seguro social (EsSalud) fue USD 3051,0 y por la atención privada fue USD 4 090,7. La pérdida media anual según el número de atenciones por hidatidosis humana no complicada fue USD 24 542,0 en el Minsa, USD 369 292,0 en EsSalud y USD 130 566,4 en la atención privada. Las pérdidas de productividad humana constituyen la minoría. Nuestros hallazgos indican que la hidatidosis impone una pesada carga económica en la región Junín.
\end{abstract}

Palabras clave: Hidatidosis; Análisis de costos; Equinococosis (fuente: DeCS BIREME).

\section{EVALUATION OF ECONOMIC EXPENDITURE RELATED TO HUMAN HYDATIDOSIS IN JUNÍN, PERU}

\begin{abstract}
The objective of the present study was to calculate the economic loss caused by the treatment of human hydatidosis. For this purpose, a cost analysis of the cases reported in the Junin region of Peru in 2013 was conducted. The total cost (direct and indirect) per patient assisted by the Ministry of Health (Ministerio de Salud-Minsa), the social security system (EsSalud), and private health care was 1,220, 3,051, and 4,090 US dollars (USD), respectively. The mean annual loss considering the number of health care services for uncomplicated cases of human hydatidosis was USD 24,542 in Minsa, USD 369,292 in EsSalud, and USD 130,566 in private health care. Overall, the rate of loss of human productivity was a small proportion. Our findings indicate that hydatidosis imposes a heavy economic burden in the Junin region of Peru.
\end{abstract}

Key words: Hydatidosis; Cost analysis; Echinococcosis (source: MeSH NLM).

\section{INTRODUCCIÓN}

La hidatidosis es una infección zoonótica causada por el estadio larvario del cestode Echinococcus granulosus, su huésped definitivo es el perro (Canis lupus familiaris) cuyas heces contaminan el suelo, el agua, los alimentos y el pasto; el ganado herbívoro sirve como huésped intermediario que se infecta al ingerir los pastos contaminados. El embrión hexacanto eclosiona de los huevos del parásito, penetra la pared intestinal y migra por el torrente sanguíneo a los diferentes tejidos, con preferencia del hígado y pulmones, aquí se convierten en metacestodos (quistes) (1). Las personas se infectan al ingerir los huevos a través del consumo de alimentos contaminados con las heces de un perro infectado (2).
En Perú, la prevalencia de hidatidosis varía entre 7 a 11 casos por 100000 personas, y en regiones ganaderas como Junín esta cifra alcanza entre 14 a 34 casos por 100000 habitantes ${ }^{(3)}$, a pesar que se han implementado múltiples estrategias, esta enfermedad sigue siendo un gran problema para la salud pública del Perú.

La incidencia anual quirúrgica por hidatidosis en el Perú es de 127 casos por cada 100000 personas-año; asimismo, el $1,9 \%$ de los pacientes fallecen por complicaciones posquirúrgicas a causa de esta enfermedad ${ }^{(4)}$.

La población de Perú es aproximadamente 30 millones de personas, de los cuales el $74 \%$ viven en zonas urbanas ${ }^{(5)}$, en el año 2014 el producto bruto interno fue de USD 202,6

\footnotetext{
Universidad Continental. Huancayo, Perú

Departamento de Enfermedades Infecciosas Tropicales y Dermatológicas, Hospital Daniel Alcides Carrión. Huancayo, Perú.

Sociedad Científica de estudiantes de Medicina, Universidad Continental. Huancayo, Perú.

Médico, especialista en enfermedades infecciosas y tropicales; ${ }^{\text {b }}$ estudiante de Medicina

Recibido: 28/11/2016 Aprobado: 23/08/2017 En línea: 29/09/2017
} 
mil millones y la población con algún tipo de seguro fue del $70,1 \%$ (Seguro Integral de Salud: $41,2 \%$, EsSalud: $23,1 \%$ y otros seguros $5,8 \%)^{(6)}$.

Es importante evaluar los costos que genera la atención de esta enfermedad, con el fin de valorar las inversiones económicas necesarias, mejorar la eficiencia en la toma de decisiones debido a las grandes pérdidas económicas que ocasionan los procedimientos de diagnóstico, tratamiento quirúrgico, farmacológico, gastos de hospitalización, recuperación, secuelas, muertes y pérdidas de producción. Asimismo, la evaluación económica nos brinda criterios sobre la mejor forma de aplicación de los recursos en salud. Por tal motivo, el objetivo del presente estudio es estimar el gasto económico ocasionado por la atención del paciente con hidatidosis en la región Junín durante el 2013.

\section{EL ESTUDIO}

\section{DISEÑO DE ESTUDIO}

Se realizó un estudio de evaluación económica parcial de tipo análisis de costos para obtener una estimación cuantificada de los gastos en salud, que genera la atención a los pacientes con hidatidosis. Para esto, se analizó los dos tipos de costos de enfermedad, la directa e indirecta, incluyéndose en cada una de ellas los costos tangibles e intangibles.

\section{POBLACIÓN Y SELECCIÓN DE CASOS}

Junín es una región endémica de hidatidosis; ubicada en los andes centrales del Perú, su principal fuente productiva es la actividad ganadera. El número total de casos de hidatidosis se obtuvo del reporte de la red de vigilancia epidemiológica regional del año 2013, que incluyó a los pacientes atendidos en los centros hospitalarios de referencia: Ministerio de Salud (Minsa) con 128 casos, Seguridad Social (EsSalud) con 121 casos y de dos clínicas particulares con 23 casos; reportados a la Dirección Regional de Salud de Junín.

\section{MUESTRA}

La unidad de análisis fue el paciente con diagnóstico de hidatidosis humana, atendido en cualquiera de estos tres centros hospitalarios. El tarifario institucional fue independiente a cada centro hospitalario. Se incluyeron a personas de todas las edades. El muestreo fue no probabilístico por enrolamiento consecutivo.

\section{VARIABLES}

La variable dependiente fue la hidatidosis, las variables independientes fueron los costos directos (prestaciones de atención médica y sanitaria, exámenes generales, serológicos, bacteriológicos, de imágenes, tratamiento farmacológico y quirúrgico según tipo de quistectomía)

\section{MENSAJES CLAVE}

Motivación para realizar el estudio. Es importante evaluar los costos que genera el tratamiento de la hidatidosis para mejorar la eficiencia en la toma de decisiones para la prevención.

Principales hallazgos. El costo total (directo e indirecto) por paciente atendido por el Ministerio de Salud (Minsa) fue de USD 1 220,0; por el seguro social (EsSalud) fue de USD 3051,0 y por la atención privada fue de USD 4 090,7.

Implicancias. El tratamiento de la hidatidosis impone una pesada carga económica en la región Junín y reduce la productividad por el salario anual perdido.

y los costos indirectos (honorarios profesionales dejados de percibir, pérdida de producción, cuidados del paciente por un tercero al alta hospitalaria y fallecimiento).

El horizonte temporal de los costos fue considerado según la estancia hospitalaria y el número de días de descanso médico. La determinación de los costos se realizó mediante la metodología de microcosteo por tipo de servicio prestado.

\section{ESTIMACIÓN DE COSTO}

Para el análisis de los casos confirmados de hidatidosis se consideraron los criterios de diagnóstico de la Organización Mundial de la Salud (OMS) ${ }^{(1)}$, según la detección de quistes por ecografía, radiografía, tomografía computarizada o resonancia magnética, y confirmadas mediante exámenes de ELISA, Western Blot, inmunoelectroforesis, anatomía patológica o respuesta al tratamiento, no se incluyeron los datos de los casos probables.

Se asumió que la proporción de casos de hidatidosis con cirugía presentó una distribución similar ${ }^{(9)}$, no se incluyeron los casos asintomáticos no diagnosticados, a pesar de conocer una prevalencia de 5,7\% (estudio basado en el análisis ecográfico de pacientes asintomáticos en una zona endémica) ${ }^{(10,11)}$, estos pacientes presentan pérdida de costo por productividad de $2 \%{ }^{(12)}$.

La pérdida de costo por productividad corresponde a la pérdida de tiempo productivo debido a que el trabajo de una persona infectada es menos eficiente que una persona no infectada, asumiendo como ingreso mensual el sueldo mínimo vital en Perú para el año 2013.

Para la estimación del costo del tratamiento quirúrgico de la hidatidosis, se consideró dos formas de intervención (la quistectomía hepática y la pulmonar), así como 14 días de hospitalización (en base al promedio de estancia hospitalaria). El costo en pacientes con infección múltiple (hígado más pulmón) fue considerado en forma separada. 
Nuestro estudio no ha considerado los parámetros epidemiológicos en el ganado ovino, a pesar que la pérdida de producción por infección hepática en ovejas infectadas es de 10 al $20 \%{ }^{(13)}$ y la prevalencia de hidatidosis en el ganado ovino en zonas endémicas de Perú es $10 \%{ }^{(14,15)}$.

\section{ANÁLISIS ESTADÍSTICO}

Los precios fueron obtenidos de tres fuentes, según su vigencia al 31 de diciembre de 2013: a) La guía para el uso del catálogo de servicios del Ministerio de Economía y Finanzas de Perú (7); b) La unidad de finanzas de un hospital nacional del Seguro Social (EsSalud) ${ }^{(8)}$, y c) La oficina administrativa de dos clínicas particulares, considerándose el costo promedio.

El tipo de cambio al 31 de diciembre de 2013 fue un dólar americano por tres soles ( 1 USD $=3$ PEN). Los datos fueron incorporados en una base en Microsoft Excel y en el programa estadístico STATA versión 11,0.

El proyecto de investigación fue aprobado por el Comité de Ética en Investigación del Hospital Docente Clínico Quirúrgico "Daniel Alcides Carrión" de Huancayo. Toda la información fue manejada manteniendo la confidencialidad de los pacientes.

\section{RESULTADOS}

Se identificaron 272 casos de hidatidosis en la región Junín durante el año 2013, atendidos en los tres centros hospitalarios de referencia (Minsa, EsSalud y atención privada); de ellos, $68,1 \%$ fueron de sexo femenino; la edad promedio fue 45 años; el órgano más afectado fue el hígado $(60,6 \%)$, y la estancia hospitalaria promedio fue de 14 días. El $74,6 \%$ de los pacientes fueron sometidos a tratamiento quirúrgico; el $1,8 \%$ de los pacientes fueron nuevamente hospitalizados por complicaciones debido a la cirugía; el 25,4\% recibieron tratamiento farmacológico, y la proporción de pacientes fallecidos fue 1,1\% (Tabla 1).

El costo directo por exámenes auxiliares de diagnóstico se estimó de acuerdo al valor unitario por el promedio del número de exámenes realizados, obteniendo un resultado de USD 105,1 en el Minsa; de USD 157,7 en EsSalud, y de USD 156,0 en la atención privada. El costo del tratamiento quirúrgico, farmacológico, así como todo el procedimiento para realizar estas estimaciones, se describen en la Tabla 2.

Para la estimación del costo producido por licencias médicas, se consideró el sueldo mínimo vital de USD 250 para los pacientes atendidos por el Minsa; se consideró el promedio de ingreso mensual de USD 615 para los pacientes atendidos por EsSalud y se consideró el promedio de ingreso mensual de USD 1136,3 para los pacientes atendidos en la atención privada ${ }^{(16)}$. En cada caso se obtuvo los costos de USD 8,3; USD 20,5 y USD 37,9 por día, respectivamente.

El costo por déficit de producción fue calculado en base al producto bruto interno (PBI) per cápita del año 2013 (USD 2180,3), para nuestro análisis dividimos el PBI entre 365 días y obtenemos un valor de USD 5,9 por día; a su vez, lo multiplicamos por 14 (días no trabajados debido al tiempo de hospitalización promedio) y obtenemos un valor de USD 82,6.

Los pacientes que egresan del hospital requieren atención domiciliaria por un cuidador (familiar o profesional de salud contratado), para determinar el costo que genera los cuidados postalta, se dividió el salario mínimo vital de USD 250,0 entre 30 días, el resultado fue multiplicado por el número promedio de días de descanso médico (el cual fue asignado por su médico tratante de acuerdo al tiempo que requiere el paciente para su incorporación laboral), es decir, 30 días, y se obtuvo un valor de USD 175,0.

El costo directo total que generó la atención por paciente con diagnóstico de hidatidosis fue de USD 637 en el Minsa, USD 2103 en EsSalud, y USD 2621 en la atención privada. En general, los costos directos de la atención por hidatidosis en el Minsa fueron cuatro veces menos que la atención privada.

Las pérdidas económicas por productividad (costo indirecto) constituyen la minoría (USD 750) de la estimación del

Tabla 1. Parámetros usados para estimar las pérdidas económicas por hidatidosis, Junín 2013

\begin{tabular}{|c|c|c|}
\hline Parámetros & $n=272$ & $\%$ \\
\hline Edad, media (rango) & $45(2-91)$ & \\
\hline \multicolumn{3}{|l|}{ Sexo } \\
\hline Masculino & 87 & 31,9 \\
\hline Femenino & 185 & 68,1 \\
\hline \multicolumn{3}{|l|}{ Localización } \\
\hline Hígado & 165 & 60,6 \\
\hline Pulmón & 101 & 37,1 \\
\hline Otros & 6 & 2,3 \\
\hline Tratamiento quirúrgico & 203 & 74,6 \\
\hline Tratamiento farmacológico & 69 & 25,4 \\
\hline $\begin{array}{l}\text { Referido a otro centro } \\
\text { hospitalario* }\end{array}$ & 26 & 9,6 \\
\hline $\begin{array}{l}\text { Reingreso por } \\
\text { complicaciones }{ }^{\dagger}\end{array}$ & 5 & 1,8 \\
\hline Desenlace fatal & 3 & 1,1 \\
\hline
\end{tabular}


Tabla 2. Estimación del costo directo en dólares para el tratamiento de quiste hidatídico sin complicaciones por paciente, Junín 2013

\begin{tabular}{|c|c|c|c|c|c|c|c|}
\hline \multirow[t]{2}{*}{ Prestaciones } & \multicolumn{3}{|c|}{$\begin{array}{c}\text { Costo unitario según tipo de centro } \\
\text { hospitalario }\end{array}$} & \multirow[t]{2}{*}{$\begin{array}{l}\text { Promedio } \\
\text { exámenes* }\end{array}$} & \multicolumn{3}{|c|}{$\begin{array}{c}\text { Costo total según tipo de centro } \\
\text { hospitalario }\end{array}$} \\
\hline & Minsa ${ }^{(6)}$ & EsSalud (7) & Privado & & Minsa & EsSalud & Privado \\
\hline Atención médica hospital/día & 3,6 & 12,0 & 33,3 & & $49,9 \|$ & $168,0 \|$ & $467,0 \|$ \\
\hline Día-cama clínico quirúrgica & 9,0 & 69,7 & 50,0 & & $126,0 \|$ & $975,3 \|$ & $700,0 \|$ \\
\hline Quiste hidatídico hepático & 184,3 & 443,0 & 1000,0 & 0,3 & 60,8 & 146,2 & 330,0 \\
\hline Quiste hidatídico pulmonar & 213,3 & 717,3 & 1333,3 & 0,3 & 55,5 & 186,5 & 346,7 \\
\hline \multicolumn{8}{|l|}{ Exámenes generales } \\
\hline Exámenes de laboratorio ${ }^{\dagger}$ & 39,0 & 83,0 & 156,0 & 1,9 & 74,2 & 157,7 & 296,4 \\
\hline Prequirúrgico ${ }^{\ddagger}$ & 26,8 & 42,7 & 113,3 & 0,6 & 16,1 & 25,6 & 68,0 \\
\hline \multicolumn{8}{|l|}{ Serología } \\
\hline Elisa & 9,3 & 10,3 & 16,7 & 0,8 & 7,5 & 8,3 & 13,3 \\
\hline Western Blot & 15,3 & 39,7 & 23,3 & 0,3 & 4,6 & 11,9 & 7,0 \\
\hline Anatomía patológica & 13,0 & 69,0 & 23,3 & 0,6 & 7,8 & 41,4 & 14,0 \\
\hline \multicolumn{8}{|l|}{ Bacteriológico } \\
\hline Cultivo y antibiograma & 6,9 & 15,0 & 16,7 & 0,1 & 0,7 & 1,5 & 1,7 \\
\hline \multicolumn{8}{|l|}{ Imágenes } \\
\hline Radiografía simple & 6,3 & 13,3 & 16,7 & 1,6 & 10,1 & 21,3 & 26,7 \\
\hline Ecografía & 6,8 & 22,7 & 20,0 & 1,7 & 11,5 & 38,5 & 34,0 \\
\hline $\begin{array}{l}\text { Tomografía computarizada } \\
\text { contrastada } \S\end{array}$ & 157,7 & 170,3 & 200,0 & 0,3 & 47,3 & 51,1 & 60,0 \\
\hline $\begin{array}{l}\text { Resonancia magnética } \\
\text { contrastada } \$\end{array}$ & 258,7 & 388,7 & 366,7 & 0,1 & 20,7 & 31,1 & 29,3 \\
\hline \multicolumn{8}{|l|}{ Tratamiento farmacológico } \\
\hline Antibioticoterapia (profilaxis) & 0,6 & 1,1 & 4,0 & 0,6 & 0,4 & 0,6 & 2,4 \\
\hline Antiparasitario (albendazol) & 22,4 & 22,4 & 56,0 & 1,0 & 22,4 & 22,4 & 56,0 \\
\hline Transfusión sanguínea & 42,3 & 112,7 & 166,7 & 0,3 & 12,7 & 33,8 & 50,0 \\
\hline \multicolumn{8}{|l|}{ Tratamiento quirúrgico } \\
\hline Quistectomía hepática & 60,0 & 190,7 & 400,0 & 0,6 & 36,0 & 114,4 & 240,0 \\
\hline Quistectomía pulmonar & 160,0 & 340,0 & 433,3 & 0,2 & 32,0 & 68,0 & 86,7 \\
\hline Total costo directo & & & & & 637,0 & 2103 & 2621 \\
\hline
\end{tabular}

Minsa: Ministerio de Salud, Essalud: Seguro Social, privado: atención privada

* Exámenes promedio por paciente.

† Exámenes de laboratorio: glucosa, urea, creatinina, hemograma, electrolitos plasmáticos, gases arteriales, perfil hepático y perfil lipídico.

‡ Prequirúrgico: grupo y factor sanguíneo, RPR, perfil de coagulación (tiempo de coagulación, sangría, tiempo de protrombina, tiempo parcial de tromboplastina, orina completa), ELISA VIH.

$\S$ Incluye gastos operativos y gastos por material médico e insumos.

|| Calculado en base a 14 días de hospitalización (estancia hospitalaria promedio)

Tipo de cambio al 31 de diciembre del 2013 ( 1 dólar $=3$ soles)

costo total, principalmente por la tardanza en la reinserción a la actividad productiva del paciente posoperado y por los salarios perdidos. El costo total (directo e indirecto) por paciente atendido es de USD 1221,0 en el Minsa; USD 3052,0 en EsSalud, y USD 5676,8 en la atención privada (Tabla 3). La pérdida económica por hidatidosis, en Junín en el año 2013 fue de USD 156 288,0 en el Minsa; USD 369 292,0 en EsSalud y USD 130 566,4 en la atención privada, valor final que se obtiene de multiplicar por el número total de pacientes con hidatidosis confirmada (128, 121 y 23 respectivamente).
La pérdida económica que genera la muerte de un paciente por hidatidosis fue estimada en base a los años perdidos por enfermedad (esperanza de vida para el año 2013 menos el promedio de edad de los afectados) y multiplicado por el ingreso mínimo mensual, obteniendo una pérdida por paciente fallecido de USD 90 600,0.

\section{DISCUSIÓN}

Este estudio intenta estimar la carga económica anual por hidatidosis en los pacientes de la región Junín de Perú, 
Tabla 3. Costo directo e indirecto en dólares para el tratamiento de quiste hidatídico por paciente, Junín 2013

\begin{tabular}{llcc}
\hline \multirow{2}{*}{ Tipo de costo } & \multicolumn{3}{c}{$\begin{array}{c}\text { Costo total según tipo de } \\
\text { centro hospitalario }\end{array}$} \\
\cline { 2 - 3 } & Minsa & EsSalud & Privado \\
\hline $\begin{array}{l}\text { Costo indirecto } \\
\quad \text { Por licencia médica* }\end{array}$ & 250,0 & 615,0 & 1136,3 \\
\hline $\begin{array}{l}\text { Por pérdida de } \\
\quad \text { producción } \\
\quad \text { (PBI per cápita/día) }{ }^{\dagger}\end{array}$ & 83,3 & 83,3 & 83,3 \\
$\quad \begin{array}{c} \\
\quad \text { Cuidados del paciente } \\
\text { postalta hospitalaria }{ }^{\ddagger}\end{array}$ & 250,0 & 250,0 & 250,0 \\
\hline $\begin{array}{l}\text { Total costo indirecto } \\
\text { Costo directo }\end{array}$ & 583,3 & 948,3 & 1469,7 \\
\hline $\begin{array}{l}\text { Costo total por paciente } \\
\text { (directo + indirecto) }\end{array}$ & 1220,0 & 3051,3 & 4090,7 \\
\hline
\end{tabular}

Minsa: Ministerio de Salud, Essalud: Seguro Social, Privado: Atención privada

* Se estimó de acuerdo al salario mínimo vital (USD 250,0) para los pacientes del Minsa, ingreso promedio para los pacientes de EsSalud (USD 615) y de atención privada (USD 1136,0); dividido entre 30 días, multiplicado por los días de descanso médico (promedio 30 días)

† Pérdida de producción: PBI per cápita (USD 2180,3) dividimos entre 365 días y multiplicado por los 14 días no trabajados

$\ddagger$ Calculado de acuerdo al salario mínimo vital (USD 250,0), dividido por 30 días, multiplicado por los días de descanso médico (promedio 30 días) Tipo de cambio al 31 de diciembre de 2013 ( 1 USD =3 PEN)

proporcionando datos para la asignación de recursos en la prevención de esta enfermedad.

A pesar de que, clásicamente, el $10 \%$ de los pacientes con hidatidosis son tratados farmacológicamente debido a que no pueden operarse ${ }^{(17)}$, nuestro estudio encontró que este número se ha incrementado, quizá porque existen nuevas recomendaciones que sugieren solo tratamiento farmacológico para los quistes hepáticos únicos menores de $5 \mathrm{~cm}$.

La estimación del costo producido por el tratamiento quirúrgico por paciente, muestra una gran diferencia entre el sector público y el privado, a pesar de esta desigualdad, nuestra estimación se asemeja a datos publicados por otros autores cuya proporción de costos en estos sectores es 1 a $3^{(18)}$. Asimismo, el resultado del gasto ocasionado por la atención al paciente con hidatidosis es similar a lo reportado por Pérez León en un estudio realizado el año $2005^{(19)}$. Nuestros resultados indican que la hidatidosis incrementa los costos indirectos y reduce la productividad por el salario anual perdido.

Una limitación de este estudio es que hemos utilizado el promedio nacional de ingreso económico (salario mínimo vital) para estimar los costos de utilidad pérdida, no obstante, este no refleja el valor de la "mano de obra" del empleador informal o no regulado (trabajador independiente); asimismo, no se estimó la carga social a cargo del paciente ${ }^{(20)}$. Además, el costo de la intervención quirúrgica se basó en el promedio de cada operación, según el tipo de quiste (hepático y pulmonar); los pacientes que fueron referidos a otros centros de mayor complejidad tuvieron mayores gastos; sin embargo, solo se consideró el costo de la cirugía y de la referencia, subestimándose los costos del acompañante (familiar). No obstante, no creemos que esto pueda resultar en una diferencia importante en los costos globales.

La implicancia económica que genera el fallecimiento de un paciente con hidatidosis es alta, generalmente porque son jóvenes y se encuentran en edad productiva, esta pérdida económica es 50 veces más elevada que los costos generados por la atención privada, reflejando la necesidad de implementar programas de control y prevención de esta enfermedad en la región Junín ${ }^{(18)}$.

Nuestros hallazgos indican que la hidatidosis impone una pesada carga económica en Junín, también hacemos hincapié en la importancia de reforzar las medidas de control actual para reducir las tasas de infección en los humanos.

Contribuciones de autoría: RM y TC participaron en el diseño del estudio, supervisión de la recolección de información, análisis e interpretación de datos. RM escribió el borrador del artículo y procesamiento de la información. Todos los autores revisaron críticamente el manuscrito y aprobaron la versión a publicar.

Fuentes de financiamiento: RM recibió fondos de la dirección de investigación de la Universidad Continental para el desarrollo de este estudio.

Conflictos de interés: los autores declaran no tener conflictos de interés en la publicación de este artículo.

Agradecimientos: a los doctores Percy Mayta, Cristian Diaz y Christian Mejía por la revisión del artículo.

\section{REFERENCIAS BIBLIOGRÁFICAS}

1. Eckert J, Gemmell MA, Meslin FX, Pawlowski ZS. WHO/OIE Manual on echinococcosis in humans and animals: a public health problem of global concern [Internet]. Paris: OIE- OMS; 2001. p. 100-141. [citado 10 octubre de 2016].
Disponible en: http://apps.who.int/iris/ bitstream/10665/42427/1/929044522X. pdf

2. Sánchez E, Cáceres O, Náquira C, Garcia D, Patiño G, Herrera $S$ et al. Molecular characterization of Echinococcus granu- losusfrom Peru by sequencing of the mitochondrial cytochrome $\mathrm{C}$ oxidase subunit 1 gene. Mem Inst Oswaldo Cruz. 2010; 105(6):806-10.

3. Guerra Montero L, Ramírez Breña MC. Hidatidosis humana en el Perú. Apunt 
cienc soc. 2015;05(1):94-101.

4. Rodriguez T. Estudio epidemiológico de la hidatidosis reportado durante el período 1975-1986 en los principales hospitales del área metropolitana de Lima. [Tesis]. Lima: Facultad de Medicina Veterinaria, Universidad Nacional Mayor de San Marcos; 1990.

5. INEI: el $74 \%$ de los peruanos vive en zonas urbanas y cada vez serán más. El Comercio (Lima). 29 de febrero de 2012 [citado el 20 de junio del 2016]. Disponible en: http://elcomercio.pe/economia/peru/ inei-74-peruanos-vive-zonas-urbanas-cada-vez-seran-mas-noticia-1380922

6. Grupo Banco Mundial. Informe anual 2015. Washington, DC: Banco Mundial; 2015. [citado el 20 de febrero de 2016]. Disponible en: http://documentos.bancomundial.org/curated/ es/684241468187780194/pdf/99910WBAR-Box393213B-PUBLIC-disclosed-10-2-15-SP.pdf

7. Propuesta Tarifario de Tomografías, Resonancias y otros [Internet]. Lima: Ministerio de Salud; 2014. [citado $10 \mathrm{de}$ febrero de 2016]. Disponible en: http:// www.sis.gob.pe/ipresspublicas/nuevotarifario.html

8. Resolución $\mathrm{N}^{\circ}$ 12-GCAS-ESSALUD-2010, que aprueba el catálogo de tarifas de prestaciones en salud a terceros no asegurados. EsSalud; 2010.

9. Zuñiga Mera R. Hidatidosis en el Valle del Mantaro, un problema de salud pública y quirúrgica, 2014. Apunt cienc soc. 2015;05(1):102-10. Doi: 10.18259/ acs. 2015016 .
10. Moro PL, Budke CM, Schantz PM, Vasquez J, Santivañez SJ, Villavicencio J. Economic Impact of Cystic Echinococcosis in Peru. PLoS Negl Trop Dis. 2011;5(5):e1179. doi: 10.1371/journal. pntd.0001179.

11. Moro PL, McDonald J, Gilman RH, Silva B, Verastegui M, Malqui V, et al. Epidemiology of Echinococcus granulosus infection in the central Peruvian Andes. Bull World Health Organ. 1997;75(6):553-61.

12. Torgerson PR, Carmona C, Bonifacino R. Estimating the economic effects of cystic echinococcosis: Uruguay, a developing country with upper-middle income. Ann Trop Med Parasitol. 2000;94(7):703-13.

13. Majorowski MM, Carabin H, Kilani M, Bensalah A. Echinococcosis in Tunisia: a cost analysis. Trans R Soc Trop Med Hyg. 2005;99(4):268-78. doi: 10.1016/j.trstmh.2004.06.011.

14. Ministerio de Salud. Programa Nacional Control de Zoonosis. Archivos de los seminarios nacionales en zoonosis y enfermedades transmitidas por alimentos. Lima: Ministerio de Salud; 1989.

15. Benner C, Carabin H, Sánchez-Serrano LP, Budke CM, Carmena D. Analysis of the economic impact of cystic echinococcosis in Spain. Bull World Health Organ. 2010; 88(1):49-57. doi: 10.2471/ BLT.09.066795.

16. Ministerio del Trabajo y Promoción del Empleo. Informe Anual del empleo en el Perú, 2014. Lima: Ministerio del Trabajo y Promoción del Empleo; 2015. [citado el 15 de enero del 2016]. Disponible en: http://www.trabajo.gob.pe/archi-
vos/file/estadisticas/peel/enaho/INFORME_ANUAL_EMPLEO_ENAHO_2014.pdf

17. EsSalud, Reporte anual epidemiológico 2014. Lima: Gerencia Central de Prestaciones de Salud, Oficina de Planeamiento e Inteligencia Sanitaria; 2015. [citado el 2 de febrero de 2016] Disponible en: http://www.essalud.gob.pe/\%20noticias/repidemiologico_se28.pdf

18. Venegas J, Espinoza S, Sánchez G. Estimación del impacto económico de la equinococosis quística en Chile y análisis de las posibles causas que han dificultado su erradicación. Rev Med Chil. 2014;142(8):1023-33. doi: 10.4067/ S0034-98872014000800010.

19. Pérez León CR. Proyecto de control de hidatidosis en el Perú por vigilancia epidemiológica. [Tesis doctoral]. Lima: Facultad de Medicina Humana, Universidad Nacional Mayor de San Marcos; 2007. [citado el 3 de febrero de 2016]. Disponible en: http://cybertesis.unmsm. edu.pe/bitstream/cybertesis/1344/1/Perez_lc\%282\%29.pdf

20. Esperanza de vida al nacer [Internet]. Lima: Instituto Nacional de Estadística e Informática. [citado el 12 de marzo de 2016]. Disponible en: https://www. inei.gob.pe/media/MenuRecursivo/ publicaciones_digitales/Est/Lib0015/ cap-59.htm

Correspondencia: Raúl Montalvo Otivo. Dirección: Av. Carrión 1550. Huancayo, Perú. Teléfono: (064) 992406768

Correoelectrónico:otivo3@hotmail.com

\section{Nuestros artículos se encuentran indizados en:}

\section{SCEPUS}

wWw.scopus.com 